\title{
Erratum to: Unresectable Hepatocellular Carcinoma: Radioembolization Versus Chemoembolization: A Systematic Review and Meta-analysis
}

\author{
Laila Lobo $^{1}$ - Danny Yakoub ${ }^{1}$ Omar Picado ${ }^{1}$ - Caroline Ripat $^{1} \cdot$ Fiorella Pendola $^{1}$. \\ Rishika Sharma $^{1}$ - Rana ElTawil ${ }^{1}$ - Deukwoo Kwon ${ }^{2}$ - Shree Venkat ${ }^{3}$. \\ Loraine Portelance $^{4} \cdot$ Raphael Yechieli ${ }^{4,5}$
}

Published online: 25 May 2017

(C) Springer Science+Business Media New York and the Cardiovascular and Interventional Radiological Society of Europe (CIRSE) 2017

\section{Erratum to: Cardiovasc Intervent Radiol (2016) 39:1580-1588 DOI 10.1007/s00270-016-1426-y}

Corrections to the last section ("Survival") on page 1582: Line: 7

Word: 3

TARE should be changed to TACE

Word: 9

TACE should be changed to TARE

Line: 10

Word: 4

TARE should be changed to TACE

The online version of the original article can be found under doi:10. 1007/s00270-016-1426-y.

Raphael Yechieli

ryechieli@med.miami.edu

1 Division of Surgical Oncology at Department of Surgery, Sylvester Comprehensive Cancer Center, University of Miami - Miller School of Medicine, Miami, FL, USA

2 Department of Biostatistics and Bioinformatics, Sylvester Comprehensive Cancer Center, University of Miami - Miller School of Medicine, Miami, FL, USA

3 Department of Radiology, Sylvester Comprehensive Cancer Center, University of Miami - Miller School of Medicine, Miami, FL, USA

4 Department of Radiation Oncology, Sylvester Comprehensive Cancer Center, University of Miami - Miller School of Medicine, Miami, FL, USA

5 Department of Radiation Oncology, Jackson Memorial Hospital/Sylvester Comprehensive Cancer Center, University of Miami - Miller School of Medicine, 1475 NW 12th Ave, Suite 1500, Miami, FL 33136, USA
Line: 11

Word: 2

TACE should be changed to TARE

Line: 13

Word: 6

TARE should be changed to TACE

The complete corrected section appears below.

Survival

Survival information was extracted from the five studies. This included 284 patients undergoing TACE and 269 patients undergoing TARE. Male to female ratio for TACE is $82: 18$ and for TARE is $77: 23$. Median age for TACE is 63 with a range of $33-88$, whereas TARE is 64 with range of 29-88. Overall survival at 1 year was $42 \%$ for TACE subjects compared to $46 \%$ for TARE. Statistically there was no difference noticed between 2 modalities $(\mathrm{RR}=0.93,95 \%$ CI $0.81-1.08, p=0.33)$. At 2 years more TACE patients were alive than those that received TARE (27 vs. 18\%) the difference of which was statistically significant $\quad(\mathrm{RR}=1.36,95 \% \quad \mathrm{CI} \quad 1.05-1.76$, $p=0.02$ ). At 3 years more TACE patients survived (14 vs. $8 \%$ ) yet no statistically significant difference was noted $(\mathrm{RR}=1.27,95 \%$ CI $0.88-1.84, p=0.20)$. At 4 years subjects alive from both TACE and TARE were $4 \%$ with no statistically significant difference in survival $(\mathrm{RR}=1.64,95 \% \mathrm{CI} 0.80-3.34, p=0.17)$. At 5 years only $1 \%$ of subject population was alive from both TACE and TARE treatment modalities. There was minimal heterogeneity among studies $(p>0.05)$. Disease-specific mortality $(\mathrm{RR}=1.58,95 \%$ CI $0.49-5.10, p=0.44)$ did not show difference between studies but high heterogeneity was noted $(\pi 2=0.6462, p=0.0015, I 2=90 \%)$. 